\title{
A Ground-Truthed Mathematical Character and Symbol Image Database
}

\author{
Masakazu Suzuki*, Seiichi Uchida** and Akihiro Nomura*** \\ * Faculty of Mathematics, \\ ** Faculty of Information Science and Electrical Engineering, \\ $* * *$ Graduate School of Mathematics, \\ Kyushu University, 6-10-1 Hakozaki, Higashi-ku, Fukuoka-shi, 812-8581 Japan
}

\begin{abstract}
This paper describes the specifications for our ground-truthed mathematical character and symbol image database, called InftyCDB-1. The ground-truth of each character is composed of type, font, quality (touched/broken) and link (relative position), etc. The database includes all the characters and symbols of 467 pages of 30 articles on mathematics, and is organized so that it can be used as word image database or as mathematical formula image database. InftyCDB-1 is a public database that is freely usable for research and development purposes.
\end{abstract}

\section{Introduction}

In this paper, we report the specifications for our groundtruthed mathematical character and symbol image database, called InftyCDB-1, which is freely usable for research and development purposes. The ground-truth of each character is composed of type, font, quality (touched/broken) and link (relative position), etc. The database includes all the characters and symbols of 467 pages of 30 articles on mathematics, and is organized so that it can be used as a word image database or as a mathematical formula image database. Thus, the database can be used, for example, in the following ways for researches:

- development and evaluation of character and scientific symbol recognition,

- development and evaluation of mathematical formula recognition,

- analysis of words in mathematical documents.

Since all the character images that appear in the page images are included in the database, users can get training data or test data for character/symbol recognition. For all the special mathematical symbols in the database, their own code and symbol name have been carefully attached. Since each alpha-numeric character in the database has its font attributes such as italic/upright, bold or not, the database can be used for evaluations of font distinction ability in character recognition.

The image data are stored separated into word or math formula units and arranged in alphabetic order independent of the content of papers. No whole page image is included in the database to avoid copyright problems.

Hereafter, the term character means not only ordinary characters (e.g., "A"), but also math symbols (e.g., "+"), unless otherwise noted. The term category means the finest level of character classification and the term type means a set of categories having a similar property. For example, "A", "B" and "C" are three categories belonging to the same type (Roman). In contrast, "A"(Roman), " $A$ "(italic), “A $\mathcal{A}$ "(calligraph), "A "(blackboard bold), " $\mathfrak{A}$ " (German), and " $\mathcal{A}$ " (script) are six categories belonging to different types. Each character belongs to either the text region or the math region. The math region includes not only numbered equations but also in-line math formulae. Note that many in-line math formulae are composed of a single character, such as " $x$ " in the sentence "The variable $x$ denotes ...".

\section{Outline of database}

\subsection{Data collection}

The documents contained in the database are 30 English articles on pure mathematics (published $1970 \sim 2000$ ). The numbers of pages, characters, words and math expressions in the database are 467, 688,570, 108,914 and 21,056, respectively. For a quantitative analysis of the database, see $[1]^{1}$. This database is larger than past databases for research on math-OCR (e.g., about 15,000 characters in [2], about 10,000 characters in [3]). Note that matrices, tables, and figures are excluded from the database.

All pages were scanned in 600 dpi and binarized automatically by the same commercial scanner (RICOH Imagio Neo 450 ). The quality of the resulting page images varies

\footnotetext{
${ }^{1}$ There are slight differences between the table1 below and the table in [1] because some errors were found after submission of the paper [1] and corrected
} 
Table 1. Characters in the database.

\begin{tabular}{|c|c|c|c|c|c|c|c|c|c|}
\hline \multirow[t]{2}{*}{ type } & \multirow[t]{2}{*}{ font } & \multirow{2}{*}{$\begin{array}{l}\text { category } \\
\text { examples }\end{array}$} & \multirow{2}{*}{$\begin{array}{r}\text { \#predefined } \\
\text { categories }\end{array}$} & \multicolumn{2}{|c|}{ text region } & \multicolumn{2}{|c|}{ math region } & \multicolumn{2}{|r|}{ total } \\
\hline & & & & \#cat. & \# char ( & \#cat. & \#char ( & \#cat. & \#char ( \\
\hline בaccent & & & $\overline{13}$ & $\overline{11}$ & 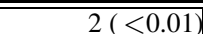 & $\overline{77}$ & 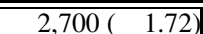 & $\overline{\overline{7}}$ & $\begin{array}{|ll|}2,702(1) & 0.39)\end{array}$ \\
\hline arrow & & $\leftarrow \leftrightarrow \leftarrow \nwarrow$ & 16 & 1 & $3(<0.01)$ & 7 & $\begin{array}{ll}1,103(0.70) \\
\end{array}$ & 7 & $\begin{array}{ll}1,106(0.16) \\
\end{array}$ \\
\hline big symbol & & $\sum 1$ & 18 & 0 & $0(0.00)$ & 11 & $2,458(1.57)$ & 11 & $2,458(0.36)$ \\
\hline blackboard bold & & $\overline{A B C D E F}$ & 26 & 0 & $0(0.00)$ & 9 & $427(0.27)$ & 9 & $427(0.06)$ \\
\hline calligraphic & & $\mathcal{A} \mathcal{B C} \mathcal{D} \mathcal{E} \mathcal{F}$ & 26 & 0 & $0(0.00)$ & 19 & $592(0.38)$ & 19 & $592(0.09)$ \\
\hline \multirow[t]{2}{*}{ German } & Upright & $\overline{\mathfrak{A} \mathfrak{B} \mathfrak{C} \mathfrak{a} \mathfrak{b} \mathfrak{c}}$ & 52 & 0 & $0(0.00)$ & 25 & $1,041(0.66)$ & 25 & $1,041(0.15)$ \\
\hline & Bold & $\overline{\mathfrak{A} \mathfrak{B C} \mathfrak{a} \mathfrak{b c}}$ & 52 & 0 & $0(0.00)$ & 0 & $0(0.00)$ & 0 & $0(0.00)$ \\
\hline \multirow[t]{4}{*}{ Greek } & Upright & $\overline{\Gamma \Delta \Theta}$ & 11 & 0 & $0(0.00)$ & 10 & $2,148(1.37)$ & 10 & $2,148\left(\begin{array}{ll}0.31) \\
\end{array}\right.$ \\
\hline & Italic & $\alpha \beta \gamma$ & 29 & 5 & $19(<0.01)$ & 23 & $10,618(6.76)$ & 23 & $10,637(1.54)$ \\
\hline & Bold & $\Gamma \Delta \Theta$ & 11 & 0 & $0(0.00)$ & 1 & $3(<0.01)$ & 1 & $3(<0.01)$ \\
\hline & Italic Bold & $\alpha \beta \gamma$ & 29 & 0 & $0(0.00)$ & 5 & $31(0.02)$ & 5 & $31(<0.01)$ \\
\hline \multirow[t]{4}{*}{ extended Latin } & Upright & $\overline{\mathrm{A} Æ \mathrm{è}}$ & 182 & 30 & $392(0.07)$ & 2 & $3(<0.01)$ & 30 & $395(0.06)$ \\
\hline & Italic & $\bar{A}$ AÈे & 182 & 9 & $55(0.01)$ & 2 & $10(0.01)$ & 10 & $65(0.01)$ \\
\hline & Bold & $\overline{\mathbf{A} / \mathbf{E} \grave{\mathbf{e}}}$ & 182 & 4 & $6(<0.01)$ & 0 & $0(0.00)$ & 4 & $6(<0.01)$ \\
\hline & Italic Bold & $\bar{A} \not E \grave{e}$ & 182 & 0 & $0(0.00)$ & 0 & $0(0.00)$ & 0 & $0(0.00)$ \\
\hline \multirow[t]{4}{*}{ numeric } & Upright & 012 & 10 & 10 & $12,018(2.26)$ & 10 & $15,294$ ( 9.74$)$ & 10 & $27,312(3.97)$ \\
\hline & Italic & 012 & 10 & 10 & $140(0.03)$ & 4 & $118(0.08)$ & 10 & $258(0.04)$ \\
\hline & Bold & 012 & 10 & 10 & $923(0.17)$ & 4 & $26(0.02)$ & 10 & $949(0.14)$ \\
\hline & Italic Bold & 012 & 10 & 0 & 0( & 0 & $0(0.00)$ & 0 & $0(0.00)$ \\
\hline operator & & $+-\times /<\&$ & 92 & 6 & $154(0.03)$ & 49 & $20,359(12.96)$ & 50 & $20,513(2.98)$ \\
\hline \multirow[t]{2}{*}{ others } & Upright & $\S @(C) \infty \forall \exists \partial$ & 42 & 10 & $2,903(0.55)$ & 15 & $1,797(1.14)$ & 20 & $4,700(0.68)$ \\
\hline & Bold & §@ @ (c) & 16 & 3 & $42(0.01)$ & 0 & $0(0.00)$ & 3 & $42(0.01)$ \\
\hline \multirow[t]{2}{*}{ parenthesis } & Upright & $($ () \{\}[ & 20 & 7 & $8,082(1.52)$ & 12 & $30,334(19.31)$ & 12 & $38,416(5.58)$ \\
\hline & Bold & ( ) \{\}[] & 20 & 2 & $112(0.02)$ & 0 & $0(0.00)$ & 2 & $112(0.02)$ \\
\hline \multirow[t]{2}{*}{ point } & Upright &, .6 & 17 & 11 & $21,599(4.06)$ & 11 & $8,443(5.41)$ & 14 & $30,042(4.36)$ \\
\hline & Bold &, .6, & 17 & 6 & $469(0.09)$ & 0 & $0(0.00)$ & 6 & $469\left(\begin{array}{ll}0.07) \\
\end{array}\right.$ \\
\hline \multirow[t]{4}{*}{ Roman } & Upright & $\mathrm{ABCabc}$ & 61 & 57 & $414,825(78.05)$ & 55 & $8,259(5.26)$ & 57 & $423,084(61.44)$ \\
\hline & Italic & $A B C a b c$ & 61 & 55 & $63,590(11.96)$ & 53 & $49,072(31.24)$ & 56 & $112,662(16.36)$ \\
\hline & Bold & A B Cabc & 61 & 56 & $6,178(1.16)$ & 13 & $538(0.34)$ & 56 & $6,716\left(\begin{array}{ll}0.98 \\
\end{array}\right.$ \\
\hline & Italic Bold & $A B C a b c$ & 61 & 0 & $0(0.00)$ & 19 & $1,508(0.96)$ & 19 & $1,508(0.22)$ \\
\hline script & & $\mathcal{A B C}$ & 52 & 0 & $0(0.00)$ & 7 & $176(0.11)$ & 7 & $176(0.03)$ \\
\hline total & & & 1,571 & 294 & $531,512(100.00)$ & 373 & $157,058(100.00)$ & 487 & $688,570(100.00)$ \\
\hline
\end{tabular}

Notes: (1) Each "Roman" and "italic" type includes nine double letters (i.e., ligatures), such as "fi".

with the quality of original print and/or copy. Several page images are noisy and include a lot of abnormal characters, such as touching or broken characters.

\subsection{Ground truth}

The ground truth for each character was attached manually by seven students in, or a graduate from, a university math department. The ground truth of each character is composed of the following attributes:

- type, category and font

- text or math region

- normal or abnormal character

- size (height and width)

- link

- location in the word or formula image

- path (folder name + file name) to the image file.

The fifth attribute, link, represents the positional relationship to the preceding character and was attached to describe the structure of a math formula (as a tree). There

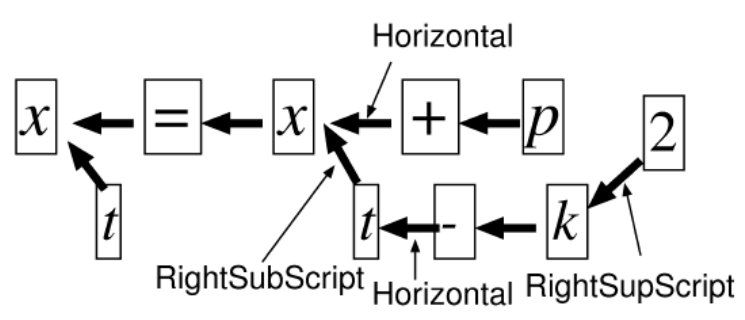

Figure 1. Link example of a math expression.

are six kinds of links: horizontal, right-superscript, rightsubscript, left-superscript, left-subscript, upper, and lower. Figure 1 shows the link structure of a formula $x_{t}=x_{t-k^{2}}+$ $p$. The sixth attribute, location, is the rectangular coordinates (left,top,right,bottom) of the character image in the corresponding word/formula image. Each character data is connected to the word/formula image data by the seventh attribute, path, and the sixth attribute, location. 


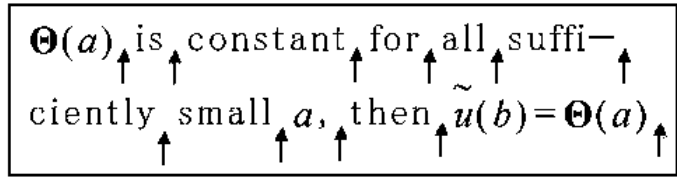

Figure 2. Word/formula segmentation.

\subsection{Word segmentation}

The segmentation of words is basically done by spacing. On the other hand, a set of consecutive math expressions in a line are unified into one formula regardless of the spacing, e.g., before and after a relative operators such as equal sign, etc. A word or a formula continued to next line is separated at the end of a line. Points (e.g. ",", “.”, etc) are included in the word/formula just before the points. Opening parenthesis are included in the next word and closing parenthesis are included in the preceding words like points. Quotation marks are treated in a similar way.

In Figure 2, the arrows show the segmentation points and the sentence is separated into 11 words/formulae in this example.

\section{Structure of database}

The database InftyCDB-1 is composed of two parts: (i) text data and (ii) image data, related to each other. Text data is a Microsoft Access or CSV-format, the user's choice, while image data are systematically named PNG files.

\subsection{Text data}

For each character, the 29 attributes listed in the Table 2 are attached:

The attribute (5) is code defined in our laboratory to distinguish character/symbol categories in the math-OCR software called InftyReader[5]. The attribute (6) is a string to read the character: e.g., "int" for " $\int$ ", "Omega" for " $\Omega$ ". The attribute (7) is "text" for text region character, and "math" for math region character. The attribute (8) is "True" (resp. "False") if the character is on the baseline (resp. in sub/super-script area). The attribute (9) (resp. (10) is "True" if the character is italic (resp. bold) font and "False" otherwise. The attribute (11) is "touched" for a touched character, "separate" for a broken character, "touch_ and_ sep" for touched and broken character, and "normal" otherwise. By using the attributes (14), (15), a user can reproduce the math tree structure for each formula.

As for the rule to define the path to the image file in the attribute (16), see 3.2 below.

Attributes (21) - (29) are the same for all the characters in a word/formula. Attribute (21) is the ID number attached to each word/formula. Attributes (22), (23) and (24) are string data that represent the corresponding word/formula
... unbounded har-

monic function $\cdots$

\section{Figure 3. SyllableAfter attribute for hyphen- ation.}

in MathML format, LaTeX format, and IML format, respectively. IML format is the XML format used in the software developed in our laboratory, math-OCR "InftyReader" and an authoring tool "InftyEditor" for mathematical documents. The attribute (29) is "True" for words at the beginning of line continued by hyphenation from the word at the end of previous line, and "False" for other cases. In Fifure 3, the latter part "monic" of "harmonic" has the SyllableAfter attribute "True".

A sample of the text data for a formula

$$
\left.\frac{\mathrm{d}}{\mathrm{dt}} \mathrm{h}_{\mathrm{t} \nu, \mathrm{z}_{0}}\right|_{\mathrm{t}=0}
$$

and a word "and" is shown in Table 3 below.

\subsection{Image data}

To reduce the number of image files, the images of a same word in a same article are stored in one image file. However, italic words and upright words are stored in different image files, and Roman capital/small letter are distinguished so that, e.g., "And" "and" are stored in different image files. Formula images in an article are also grouped in a same way, when the expressions are identical. Image file names are defined as follows:

- word ... “string”(_ FontFlag)_ “number”.png

- formula ... "number of characters in the formula" _"first three characters in the formula" (_ FontFlag)_ "number".png

For example, the file names of the word "(and)" and the formula " $\alpha \leq 1$ " are "LeftPar-and-RightPar_0.png" and "3_alpha-le-1_0.png", respectively. Figure 4 shows two image files in the database having the path names “Images $¥ A c t a M \_1970 \_37 \_63 ¥ A ¥$ approaches_I_0.png" and "Images $¥ A c t a M \_1970 \_37 \_63 ¥ M A T H ¥ 1-9 ¥ 7 \_D-$ Omega-BigLeftPar_0.png”, respectively.

\section{Distribution}

The database InftyCDB-1 is made and will be maintained in M.Suzuki laboratory, Faculty of Mathematics, Kyushu University. It is freely available for research and development purposes after user registration. (http://www.inftyproject.org/). 
Table 2. List of attributes.

\begin{tabular}{|l|l|l|}
\hline & Attribute & Explanation \\
\hline$(1)$ & CharID & ID number of each character \\
\hline$(2)$ & JornalID & ID number of the article \\
\hline$(3)$ & SheetID & Page number \\
\hline$(4)$ & Type & Type name (see Table 1) \\
\hline$(5)$ & Code & Category code (OCR code) \\
\hline$(6)$ & Entity & Category name \\
\hline$(7)$ & Region & Distinction of text/math area \\
\hline$(8)$ & Baseline & Distinction of baseline/subscript \\
\hline$(9)$ & ItalicFlag & Italic flag \\
\hline$(10)$ & BoldFlag & Bold flag \\
\hline$(11)$ & Quality & Normal/touched/separate/touch_ and_sep \\
\hline$(12)$ & Width & Width of the character \\
\hline$(13)$ & Height & Height of the character \\
\hline$(14)$ & ParentCharID & Parent CharID of the link \\
\hline$(15)$ & Link & Link name \\
\hline$(16)$ & ImageName & Path (folder name + file name) to the image file including the character \\
\hline$(17)(18)(19)(20)$ & Rect & Coordinates of the character in the image file (left,top,right,bottom) \\
\hline$(21)$ & WordID & ID number of the word/formula including the cahracter \\
\hline$(22)$ & WordMathML & MathML string \\
\hline$(23)$ & WordTeX & LaTeX string \\
\hline$(24)$ & WordIML & IML string \\
\hline$(25)(26)(27)(28)$ & WordRect & Coordinates of the word/formula in the image file (left,top,right,bottom) \\
\hline$(29)$ & SyllableAfter & Flag of word continued from the prevoious line by hyphenation \\
\hline
\end{tabular}

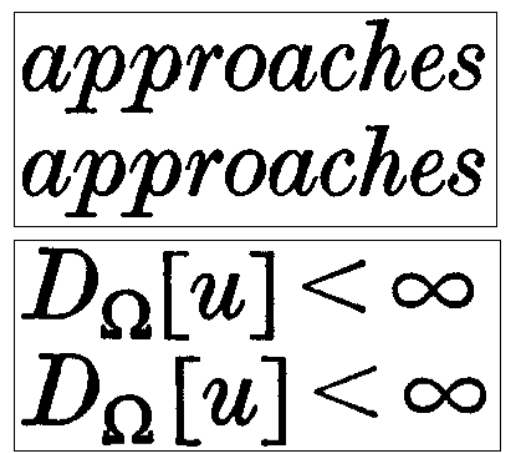

Figure 4. Example of image files

The text data part is in CSV (16.8MB) or Microsoft Access format $(26.7 \mathrm{MB})$ as the user chooses, and the image data is in PNG format (202MB). The database is delivered in CD-ROM.

\section{Conclusion}

In this paper, we described our ground-truthed mathematical character and symbol image database, called InftyCDB-1. The database consists of two parts: text data and image data that are related to each other.

The ground-truth of each character is composed of type, font, quality (touched/broken) and link information to represent the tree structure of math formula, etc. The database includes all the 688,570 characters (and symbols) of 467 pages of 30 English articles on mathematics (published $1970 \sim 2000$ ). Characters are grouped into words/formulae in the database. Total number of words and formulae in the database are 108,914 and 21,056, respectively. The database is freely usable for research, development and evaluation of math-OCRs.

\section{References}

[1] S. Uchida, A. Nomura, M. Suzuki "Quantitative Analysis of Mathematical Documents," Int. J. Doc. Anal. Recog., to appear.

[2] H.-J. Lee and J.-S. Wang, "Design of a mathematical expression understanding system," Pattern Recognition Letters, 18(3):289-298, 1997.

[3] M. Okamoto, H. Imai, and K. Takagi, "Performance evaluation of a robust method for mathematical expression recognition," Proc. ICDAR, 121-128, 2001.

[4] A. Nomura, K. Michishita, S. Uchida, and M. Suzuki, "Detection and segmentation of touching characters in mathematical expressions," Proc. ICDAR, 1:126-130, 2003.

[5] M. Suzuki, F. Tamari, R. Fukuda, S. Uchida, T. Kanahori "Infty- an integrated OCR system for mathematical documents," ACM Symposium on Document Engineering, 95-104, 2003 
Table 3. Example of the text data in the database for a formula " $\left.\frac{\mathrm{d}}{\mathrm{dt}} \mathrm{h}_{\mathrm{t} \nu, \mathrm{z}_{0}}\right|_{\mathrm{t}=0}$ " and a word "and".

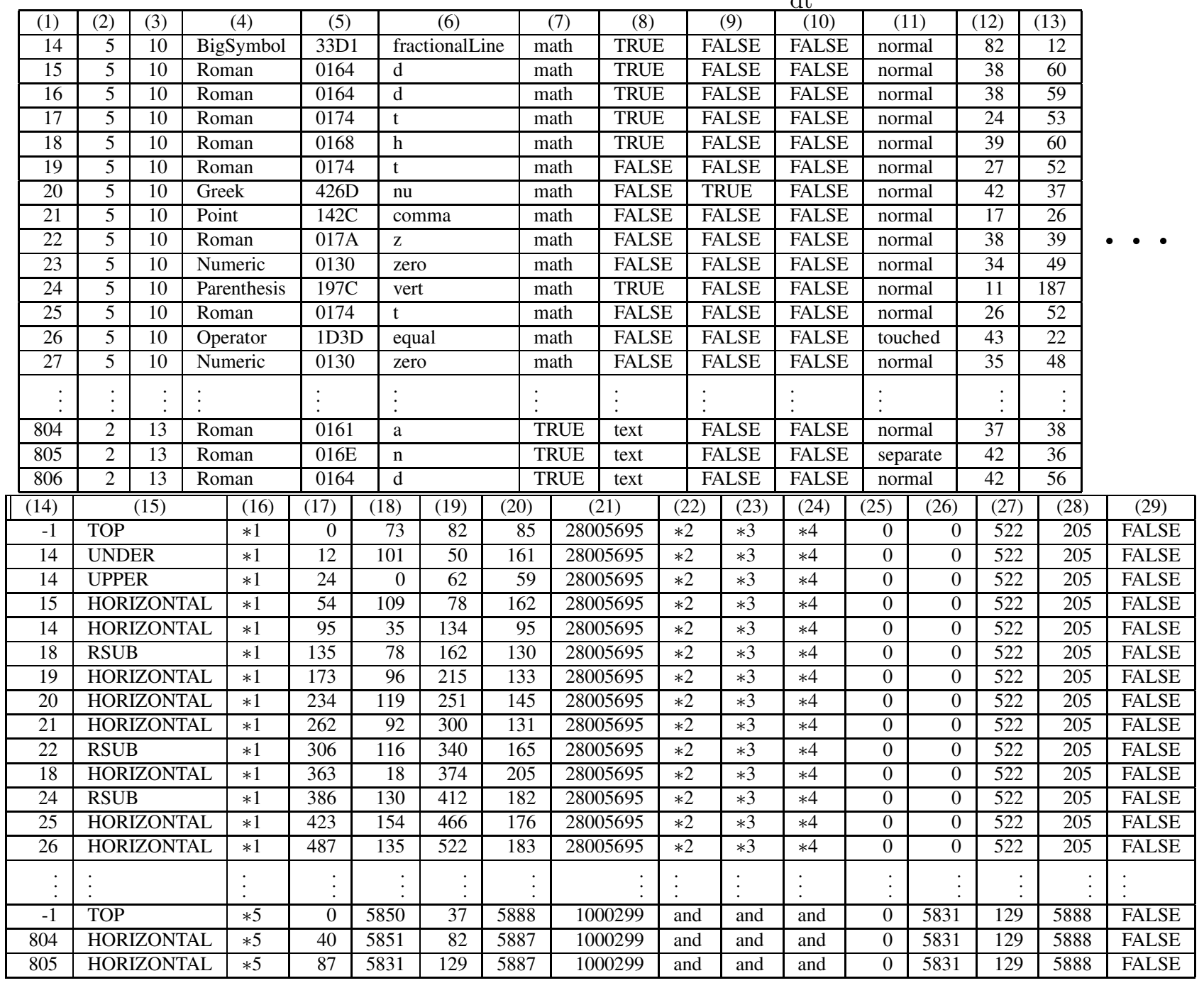

(*1) AnnMS_1971_157_173¥MATH¥10-19¥14_fractionalLine-dd_0.png, （*5) ActaM_1970_37_63¥A¥and_1.png $(* 2),(* 3),(* 4)$ : Expression of the formula $\left.\frac{\mathrm{d}}{\mathrm{dt}} \mathrm{h}_{\mathrm{t} \nu, \mathrm{z}_{0}}\right|_{\mathrm{t}=0}$ in MathML, in LaTeX and in IML respectively.

[6] J. Ha, R. M. Haralick, and I. T. Phillips, "Understanding mathematical expressions from document images," Proc. ICDAR, 956-959, 1995.

[7] Y. Eto and M. Suzuki, "Mathematical formula recognition using virtual link network," Proc. ICDAR, 762767, 2001.

\section{List of articles in the database}

- Acta Math., 124(1-2), 37-63, 1970. • ibid., 181(2), 283-305, 1998. • Ann. Sci. Ecole Norm. Sup., 4d sér, t.3, 273-284, 1970. - ibid., t.30, 367-384, 1997. • Ann. Inst. Fourier, 20(1), 493-498, 1970. • ibid., 49(2), 375-404, 1999. • Ann. Math., 91, 550-569, 1970. • Ann. Math. Studies, 66, 157-173, 1971. • Arkiv für
Matematik, 9(1), 141-163 1971. • ibid., 35(1), 185-199, 1997. - Bull. Amer. Math. Soc., 77(1), 157-159 1971. • ibid., 77(1), 160-163 1971. • ibid., 80(6), 1219-1222, 1974. • ibid., 35(2), 123-143, 1998. • Bull. Soc. Math. France, 98, 165-192, 1970. - ibid., 126, 245-271, 1998. • Invent. Math., 9, 121-134, 1970. - ibid., 138, 163-181, 1999. • J. Math. Soc. Japan, 27(2), 281288, 1975. • ibid., 27(2), 289-293, 1975. • ibid., 27(2), 497-506, 1975. • J. Math. Kyoto Univ., 11(1), 181-194, 1971. • ibid., 11(1), 373-375, 1971. • ibid., 11(2), 377-379, 1971. • Kyushu J. Math., 53, 17-36, 1999. • Math. Ann., 225(3), 275-292, 1977. - ibid., 315, 175-196, 1999. • Tohoku Math. J., 25, 317-331, 1973. • ibid., 25, 333-338, 1973. • ibid., 42, 163-193, 1990. 